\title{
SELECTION OF INFORMATIVE COLOR FEATURES FOR CLASSIFICATION OF OBJECT AREAS OF WHITE BRINED CHEESE AND MOLD
}

\author{
Zlatin Zlatev, Miroslav Vasilev, Tanya Pehlivanova \\ Trakia University, Faculty of Technics and Technologies \\ 38 Graf Ignatiev str., 8602, Yambol, Bulgaria \\ e-mail: zlatin.zlatev@trakia-uni.bg
}

\begin{abstract}
The dairy product white brined cheese is made from cows, sheep's milk or a mixture of them, which is traditional for Bulgaria. Important from a practical point of view to provide objective information about the changes occurring in the product during storage. On the contemporary level of development of science few are the publications related to changes in the surface characteristics of this product when stored under conditions not corresponding to those specified by the manufacturer. The aim of the report is to present a selection of complex color informative features describing the change of object areas of white brined cheese in storage period. Descriptive statistics is presented for 16 color components of five color models (RGB, HSV, Lab, LCH, CMYK), obtained by processing color digital images of the product. The selection of color features is made by the method "Correspondence analysis" applied to the results obtained with the distance functions. A comparative analysis of methods for classification is made. Used methods are "Cluster analysis" and "Discriminant function analysis". A set of informative color features suitable for classification is proposed.
\end{abstract}

Keywords: White brined cheese, Color features, Distance functions, Correspondence analysis, Cluster analysis, Discriminant function analysis.

\section{INTRODUCTION}

White brined cheese is a product made from cows, sheep's milk or a mixture of them, which is traditional for Bulgaria. It has undergone a physicochemical and biochemical changes due to rennet and enzymes of lactic acid organisms. Ripened cheese has moderate salinity and pronounced acidity. It is widely spread both in Bulgaria and in the culinary region of our neighboring countries $[3,8]$.

Important from a practical point of view to provide objective information about the changes occurring in the product during storage. These changes are related to the change in its surface characteristics and its physico-chemical properties.

In recent years, these changes in the properties and composition of the cheese in storage have been the subject of scientific researches $[3,8,10]$. Few are the publications related to changes in the surface characteristics of this product when stored under conditions not corresponding to those specified by the manufacturer.

Grading the cheese by its color features requires the use of precise and rapid procedures for obtaining, processing and interpreting the most informative set of features relating to the quality characteristics of the object areas on its surface. The formal representation of the object areas studied by a set of physical properties derived from the source data is used to classify their study into one of the predefined classes. Since the output data for the surface characteristics of the product being studied is formed by a system for obtaining, processing and analyzing images, the data is highly dimensional and the first phase is particularly important for the efficiency of the overall procedure and for the quality of the results obtained. 


\section{IRTITE}

Ipplied Researrohes in Technics, Technologies and Bducition

Journal of the Faculty of Technics and Technologies, Trakia University https:///sites.google.com/a/trakia-uni.bg/artte/

The first condition in the grading of features is to find a minimal description of the feature space, and the second condition is to find features that are as little as possible dependent on each other $[1,5,8]$.

The main purpose of this article is to determine the information value of color features from color models, to recognize the object areas on the surface of white brined cheese and to determine the accuracy of the classification.

\section{MATERIAL AND METHODS}

The material used in the study is white brined cheese, purchased commercially. The parameters of the cheese are:

$\checkmark$ Manufactured by BNS 15:2010, Amendment 1:2014 [2,3];

$\checkmark$ Partied: L04130217;

$\checkmark$ Content by label: Cow milk, Cheesy enzyme, Calcium dichloride, Salt, yeast;

$\checkmark$ Storage conditions by label: from $-2^{\circ} \mathrm{C}$ to $4^{\circ} \mathrm{C}$.

Samples of $10,5 \times 7,5 \mathrm{~cm}$ and $1 \mathrm{~cm}$ thick were prepared.

A Digimax 250 digital camera with serial number 34603308 was used. Digital images with resolution 1600x1200 pixels were obtained. Device settings are "default". A flash is used.

The measurement were conducted for 12 days in conditions not corresponding to those specified by the manufacturer. The storage conditions were: room temperature $20-22^{\circ} \mathrm{C}$; relative humidity $40-42 \% \mathrm{RH}$. The resulting images of changes in surface characteristics of chees during storage are presented on figure 1. Values of 16 color components of five color models RGB, HSV, Lab, LCH, CMYK are obtained.

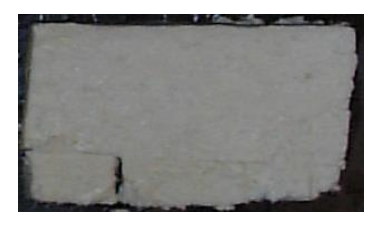

Day 3

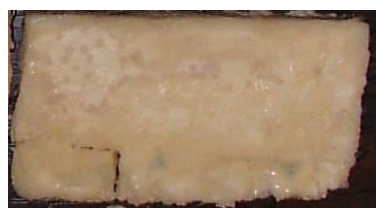

Day 7

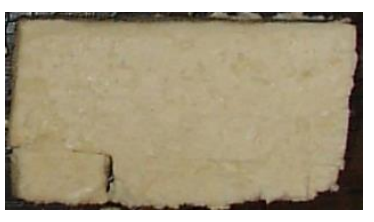

Day 4

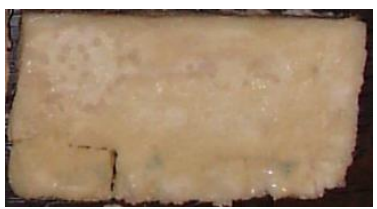

Day 8

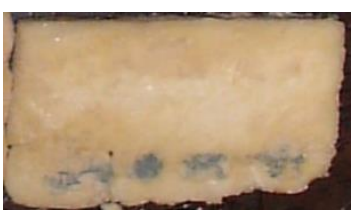

Day 11

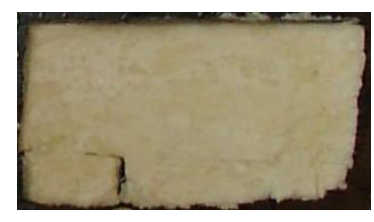

Day 5

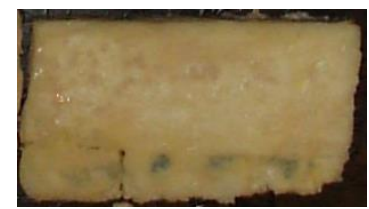

Day 9

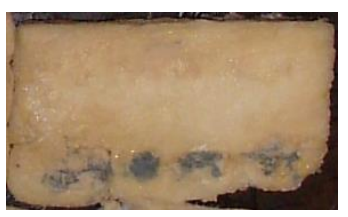

Day 12

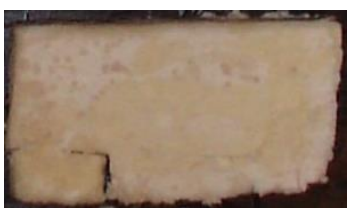

Day 6

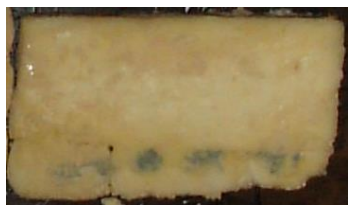

Day 10

Figure 1. Changes of the surface of cheese in storage period

In Table 1 is a description of the investigated functions of the distances between the color components. The distances used are: Mahalanobis distance, Euclidean distance, Minkowski distance, a distance of Manhattan (Cityblock), Chebyshev distance and Fisher discriminant ratio.

IRTIIE Vol. 5, No. 2, 2017 ISSN 1314-8788 (print), ISSN 1314-8796 (online), doi: 10.15547/artte.2017.02.003 


\section{IRTITE}

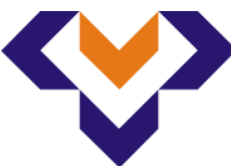

Ipplied Reseitrones in Technics, Technologies and Bductition

Journal of the Faculty of Technics and Technologies, Trakia University https://sites.google.com/a/trakia-uni.bg/artte/

The obtained data for the change of the color components during the cheese storage period represent a three-dimensional matrix of the $n_{x} k_{x} j$ type, where $D F=1 \ldots . n$ are the values of the distances determined by the represented functions; $C C=1 \ldots \mathrm{k}$ are the color components; Day $=1 . . . i$ are the days of storage. Data for each day of storage can be considered as a separate two-dimensional matrix $n_{x} k$. Figure 2 represents the used data as three dimensional matrix.

Table 1. Distance functions used in the study

\begin{tabular}{|l|c|l|}
\hline \multicolumn{1}{|c|}{ Function } & Mathematical formula & \multicolumn{1}{c|}{ Description } \\
\hline Mahalanobis & $d_{s t}{ }^{2}=\left(x_{s}-y_{t}\right) C^{-1}\left(x_{s}-y_{t}\right)^{\prime}$ & C - covariance matrix \\
\hline Euclidean & $d_{s t}{ }^{2}=\left(x_{s}-y_{t}\right)\left(x_{s}-y_{t}\right)^{\prime}$ & \multirow{2}{*}{ x and $\mathrm{y}$ - the compared vectors } \\
\cline { 1 - 2 } Cityblock & $d_{s t}=\sum_{j=1}^{n}\left|x_{s j}-y_{t j}\right|$ & \\
\hline Chebychev & $d_{s t}=\max _{j}\left\{\left|x_{s j}-y_{t j}\right|\right\}$ & max - maximum \\
\hline Fisher discriminant ratio & $d_{s t}=\frac{(\bar{x}-\bar{y})^{2}}{S D_{x}{ }^{2}+S D_{y}{ }^{2}}$ & SD - standard deviation \\
\hline
\end{tabular}

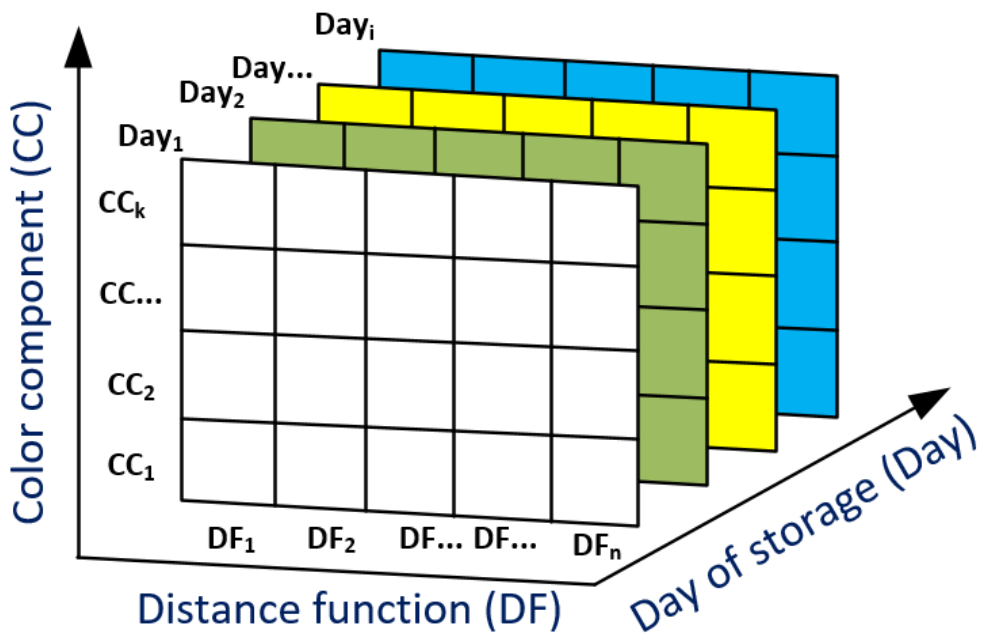

Figure 2. Three dimensional matrix of used data for color components, distance functions and days of storage

The "Correspondence analysis" method, available in the Statistica software, has been used. This analysis is a multidimensional method that enables data to be tabled by graphical interpretation, and the columns in the table can be represented as points in a small dimension space with a subsequent interpretation called the "Correspondence map" [6,9]. The classification of object areas is made by two classifiers.

$\checkmark$ Discriminant function analysis [4] which is a statistical analysis to predict a categorical dependent variable, called a grouping variable by one or more continuous or binary independent variables, called predictor variables. The "quadratic" nonlinear discriminant function is used. This function is defined as:

$$
d\left(x_{1}, x_{2}\right)=K+\left[x_{1} x_{2}\right] L+\left[\begin{array}{ll}
x_{1} & x_{2}
\end{array}\right] Q\left[\begin{array}{l}
x_{1} \\
x_{2}
\end{array}\right]
$$

where $\mathrm{K}$ is constant, $\mathrm{L}$ - linear function, $\mathrm{Q}$ - quadratic function;

IRTIIE Vol. 5, No. 2, 2017 ISSN 1314-8788 (print), ISSN 1314-8796 (online), doi: 10.15547/artte.2017.02.003 


\section{ARTIIE



Ipplied Researlohes in Technics, Technologies and Bductition

Journal of the Faculty of Technics and Technologies, Trakia University https://sites.google.com/a/trakia-uni.bg/artte/

$\checkmark$ k-means clustering. This classification method performs k-means clustering to partition the observations of the $n_{x} p$ data matrix $X$ into $k$ clusters, and returns an $n_{x} 1$ vector containing cluster indices of each observation. Rows of $X$ correspond to points and columns correspond to variables. In the study the number of replications is set to 10 replications and "sqeuclidean" separation function is used. The function Squared Euclidean distance uses each centroid as the mean of the points in cluster. It is defined by the function $d(x, c)=(x-c) \cdot(x-c)^{\prime}[7]$.

\section{RESULTS AND DISCUSSION}

Descriptive statistics of these color components of object areas with cheese are shown in Table 2. The color components are presented with their mean value, standard deviation and coefficient of variation. It can be seen that there are small values of the coefficient of variation - under $30 \%$ for most of the color components. An analysis is made of the change in the values of the color components of the color models for object areas with mold. Changes of the values of object areas with mold are not used as the primary criterion for determining the process, as such areas on the surface of the products appear visibly after day 3. The descriptive statistics of changes of the values of color components for object areas with mold are presented on Table 3.

Table 2. Descriptive statistics of color components for object areas of white brined cheese

\begin{tabular}{|c|c|c|c|c|c|c|c|c|c|c|c|c|c|c|c|c|c|}
\hline & & \multicolumn{3}{|c|}{ RGB } & \multicolumn{3}{|c|}{ HSV } & & & \multicolumn{4}{|c|}{ CMYK } \\
\hline Day & & R & $\mathbf{G}$ & B & $\mathrm{H}$ & $\mathrm{s}$ & $v$ & $\mathrm{~L}$ & $a$ & b & $\mathrm{L}$ & $\mathrm{C}$ & $H$ & c & & & \\
\hline \multirow{4}{*}{3} & mean & 131,57 & 132,42 & 129,72 & 0,24 & 0,02 & 0,52 & 140,67 & 127,31 & 129,36 & 55,16 & 1,79 & 114,26 & 44,63 & 37,52 & 44,69 & 111,54 \\
\hline & SD & 5,60 & 5,56 & 6,16 & 0,15 & 0,01 & 0,02 & 5,51 & 0,72 & 1,16 & 2,16 & 1,00 & 51,40 & 3,84 & 2,80 & 5,91 & 5,75 \\
\hline & CV & 0,04 & 0,04 & 0,05 & 0,63 & 0,57 & 0,04 & 0,04 & 0,01 & 0,01 & 0,04 & 0,56 & 0.45 & 0,09 & 0,07 & 0,13 & 0,05 \\
\hline & mean & 166,69 & 153,14 & 119,84 & 0,12 & 0,28 & 0,65 & 162,69 & 129,26 & 147,48 & 63,80 & 19,54 & 86,27 & 27,08 & 42,42 & 100,89 & 81,06 \\
\hline \multirow[t]{3}{*}{4} & SD & 9,22 & 8,59 & 8.92 & 0,01 & 0,02 & 0,04 & 8.38 & 0.97 & 1,57 & 3,29 & 1,56 & 290 & 3,78 & 4,37 & 8.18 & 876 \\
\hline & cV & 0,06 & 0,06 & 0.07 & 0,05 & 0,08 & 0.06 & 0,05 & 0.01 & 0.01 & 0.05 & 0,08 & 0,03 & 0,14 & 0,10 & 0.08 & 0,11 \\
\hline & mean & 156,37 & 143,41 & 108,14 & $\frac{1,12}{0,12}$ & 0,31 & 0,61 & 153.05 & 128,90 & 148,68 & $\frac{60.02}{60.02}$ & $\frac{10,73}{20,73}$ & 87,46 & 31,50 & 46,21 & 112,09 & $\begin{array}{r}0,88 \\
90,8\end{array}$ \\
\hline \multirow[t]{3}{*}{5} & SD & 8,11 & 8,60 & 9,52 & 0,01 & 0,03 & 0,03 & 8,22 & 1,02 & 2,05 & 3,22 & 2,04 & 2,88 & 3,46 & 5,82 & 10,60 & 7,98 \\
\hline & $\mathrm{cV}$ & 0,05 & & & 0,05 & 0,11 & 0,05 & 0,05 & 0.01 & & 0,05 & 0,10 & & 0,11 & & 0,09 & $\frac{1,00}{0,09}$ \\
\hline & mean & 163,28 & 140,42 & 114,49 & 0,09 & 0,30 & 0,64 & 153,00 & 133,97 & 145,27 & 60,00 & 18,34 & 70,56 & 27,07 & 62,21 & 98,10 & 84,50 \\
\hline \multirow[t]{2}{*}{6} & SD & 6,96 & 6,95 & 9,45 & 0,01 & 0,04 & 0,03 & 6,69 & 1,31 & 2,93 & 2,63 & 2,81 & 5,06 & 2,89 & 6,32 & 13,31 & 7,13 \\
\hline & & & & & 0,10 & 0,14 & 0,04 & 0,04 & 0,01 & & 0,04 & 0,15 & 0,07 & 0,11 & 0,10 & 0,14 & 0,08 \\
\hline \multirow{4}{*}{7} & & 176,36 & 149,45 & 118,01 & & & & 162,52 & 134,96 & 148,61 & 63,74 & 21,80 & 71,08 & 22,19 & 61,91 & 104,21 & 71,78 \\
\hline & SD & & 8,45 & 11,17 & 0,01 & 0,04 & 0,03 & & 1,32 & 3,09 & $\frac{1,14}{3.14}$ & 3,32 & 405 & $\frac{1611}{3.11}$ & 6.83 & 14,33 & 8.06 \\
\hline & CV & 0,05 & 0,06 & & 0,08 & 0,13 & 0,05 & 0,05 & 0,01 & 0,02 & 0,05 & 0,14 & 0,06 & 0,14 & 0,11 & 0,14 & 0,11 \\
\hline & mean & 175,89 & 149,91 & 117,57 & 0,09 & 0,33 & 0,69 & 162,70 & 134,46 & 148,91 & 63,80 & 21,93 & 72,75 & 22,48 & 60,12 & 105,32 & 72,31 \\
\hline \multirow[t]{2}{*}{8} & SD & 7,63 & 7,77 & 9,49 & 0,01 & 0,03 & 0,03 & 7.37 & 1,37 & 2,15 & 289 & 2.11 & 3,77 & 2.97 & 6.49 & 10.99 & 7,43 \\
\hline & $\mathrm{cV}$ & 0,04 & 0,05 & 0,08 & 0,07 & 0,10 & 0,04 & 0,05 & 0,01 & 0,01 & 0.05 & 0,10 & 0,05 & 0,13 & 0,11 & 0,10 & 0,10 \\
\hline \multirow{3}{*}{9} & mean & 156,34 & 134,83 & 87,21 & 0,11 & 0,44 & 0,61 & 146,35 & 131,51 & 156,75 & 57,39 & 28,99 & 83,04 & 31,52 & 61,12 & 146,58 & 91,39 \\
\hline & SD & $\begin{array}{l}7,17 \\
\end{array}$ & 7,70 & 9,91 & 0,01 & 0,04 & 0,03 & 7,33 & 1,41 & 2.45 & 287 & 2.47 & 2.71 & 298 & 7.03 & 12,69 & 7,43 \\
\hline & $\mathrm{cV}$ & 0.05 & 006 & 0.11 & 0.05 & 0,10 & 0,05 & 0.05 & 0.01 & 0,02 & 0.05 & 0.09 & 0.03 & 0.09 & 0,11 & 0.09 & 0.08 \\
\hline \multirow{3}{*}{10} & mean & 163,7 & 142,62 & 89,79 & 0.12 & 0,46 & 0,64 & 153,63 & 130,83 & 159,13 & 60.25 & 31,30 & 84,88 & 28.91 & 55.92 & 149.41 & 83.83 \\
\hline & SD & 10,47 & 11,93 & 15,65 & 0,01 & 0, & 0,04 & 11,21 & 162 & 3,25 & 4.40 & 3,30 & 283 & 4,20 & 9,52 & 18,74 & 10,38 \\
\hline & $\overline{c V}$ & 0,06 & 0,08 & 0,17 & 0.05 & 0,14 & $\overline{0,06}$ & 0,07 & 0,01 & 0,02 & 0,07 & 0,11 & 0,03 & 0,15 & 0.17 & 0.13 & 0,12 \\
\hline \multirow{3}{*}{11} & mean & 199,85 & 170,33 & 130,3 & 0,10 & 0,35 & 0,78 & 182,65 & 134,94 & 152,90 & 71,63 & $25,8 \mathrm{~s}$ & 74,23 & 15,09 & 54,22 & 106,15 & 48,83 \\
\hline & SD & 10,38 & 11,81 & 16 & 0,01 & 10 & 0,04 & 10,82 & 1,27 & $\begin{array}{l}3,84 \\
\end{array}$ & 4,24 & 3,82 & 3,02 & 3,13 & 7,69 & 17,98 & 10,16 \\
\hline & $\begin{array}{cl}\text { CV } \\
\text {. }\end{array}$ & 5 & 0,07 & 3 & 0,06 & 0,16 & & 0,06 & 0,01 & 0,03 & 0,06 & 0,15 & 0,04 & 0,21 & 0,14 & 0,17 & 0,21 \\
\hline \multirow{3}{*}{12} & mean & 174,4 & 142,75 & 100,91 & 0,09 & 0,43 & & 156,90 & 136,26 & 155,07 & 61,53 & 28,34 & 72,95 & 23,17 & 71,43 & 132,03 & 74,00 \\
\hline & SD & 12, & & & & & 0, & 13,70 & 1,63 & \begin{tabular}{l|l}
3,84 \\
\end{tabular} & 5,37 & 3,90 & 3,03 & 4,41 & 12,38 & 22,37 & 12,58 \\
\hline & $\overline{c V}$ & 0,07 & 0,10 & 0,19 & 0,06 & 0,17 & 0,07 & & 0,01 & & 0,09 & 0,14 & & 0,19 & 0,17 & 0,17 & 0,17 \\
\hline
\end{tabular}

Table 3. Descriptive statistics of color components for object areas with mold

\begin{tabular}{|c|c|c|c|c|c|c|c|c|c|c|c|c|c|c|c|c|c|}
\hline & & \multicolumn{3}{|c|}{ RGB } & \multicolumn{3}{|c|}{ HSV } & \multicolumn{3}{|c|}{ Lab } & \multicolumn{3}{|c|}{ LCH } & \multicolumn{4}{|c|}{ CMYK } \\
\hline day & & $\mathbf{R}$ & $\mathbf{G}$ & B & $\mathbf{H}$ & $\mathrm{S}$ & V & $\mathbf{L}$ & $a$ & b & $\mathbf{L}$ & C & $\mathrm{H}$ & C & $\mathbf{M}$ & $\bar{Y}$ & $\mathrm{~K}$ \\
\hline \multirow{3}{*}{3} & mean & 115,20 & 107,36 & 67,64 & 0,14 & 0,42 & 0,45 & 115,35 & 126,48 & 151,39 & 45,24 & 23,47 & 93,63 & 56,15 & 59,81 & 156,71 & 129,36 \\
\hline & SD & 20,94 & 18,60 & 16,91 & 0,01 & 0,06 & 0,08 & 19,54 & 1,39 & 3,29 & 7,66 & 3,31 & 3,33 & 13,30 & 9,21 & 19,68 & 20,16 \\
\hline & CV & 0,18 & 0,17 & 0,25 & 0,07 & 0,14 & 0,18 & 0,17 & 0,01 & 0,02 & 0,17 & 0,14 & 0,04 & 0,24 & 0,15 & 0,13 & 0,16 \\
\hline \multirow{3}{*}{4} & mean & 106,85 & 106,37 & 76,58 & 0,17 & 0,30 & 0,42 & 112,62 & 124,30 & 144,83 & 44,16 & 17,32 & 103,01 & 68,38 & 55,01 & 136,00 & 132,18 \\
\hline & SD & 26,99 & 25,04 & 23,21 & 0,02 & 0,10 & 0,10 & 26,75 & 1,60 & 4,24 & 10,49 & 4,17 & 6,21 & 20,28 & 15,44 & 27,03 & 26,35 \\
\hline & CV & 0,25 & 0,24 & 0,30 & 0,12 & 0,33 & 0,24 & 0,24 & 0,01 & 0,03 & 0,24 & 0,24 & 0,06 & 0,30 & 0,28 & 0,20 & 0,20 \\
\hline \multirow{3}{*}{5} & mean & 99,97 & 99,17 & 70,89 & 0,17 & 0,32 & 0,40 & 105,31 & 124,87 & 144,12 & 41,30 & 16,71 & 103,56 & 72,09 & 60,49 & 138,23 & 139,34 \\
\hline & SD & 27,31 & 27,54 & 29,05 & 0,04 & 0,19 & 0,11 & 28,89 & 2,39 & 8,56 & 11,33 & 8,33 & 13,20 & 19,40 & 20,39 & 40,76 & 29,18 \\
\hline & CV & 0,27 & 0,28 & 0,41 & 0,24 & 0,58 & 0,27 & 0,27 & 0,02 & 0,06 & 0,27 & 0,50 & 0,13 & 0,27 & 0,34 & 0,29 & 0,21 \\
\hline \multirow{3}{*}{6} & mean & 83,62 & 90,03 & 77,82 & 0,30 & 0,18 & 0,36 & 95,14 & 123,69 & 134,19 & 37,31 & 8,54 & 136,83 & 87,70 & 59,16 & 104,72 & 150,33 \\
\hline & SD & 15,77 & 16,77 & 20,29 & 0,11 & 0,15 & 0,06 & 17,72 & 2,16 & 6,61 & 6,95 & 5,70 & 28,03 & 14,05 & 14,69 & 33,50 & 17,61 \\
\hline & CV & 0,19 & 0,19 & 0,26 & 0,35 & 0,83 & 0,18 & 0,19 & 0,02 & 0,05 & 0,19 & 0,67 & 0,20 & 0,16 & 0,25 & 0,32 & 0,12 \\
\hline \multirow{3}{*}{7} & mean & 78,98 & 88,17 & 78,46 & 0,33 & 0,16 & 0,35 & 92,60 & 122,75 & 132,34 & 36,31 & 7,59 & 144,21 & 95,06 & 58,54 & 100,60 & 151,96 \\
\hline & SD & 18,84 & 17,66 & 15,96 & 0,10 & 0,08 & 0,07 & 19,06 & 2,29 & 3,95 & 7,47 & 3,12 & 25,55 & 18,62 & 13,19 & 18,71 & 18,54 \\
\hline & CV & $0,0,24$ & 0,20 & $\frac{10,20}{0,0}$ & 0,30 & 0,50 & $\begin{array}{l}0,20 \\
0,20\end{array}$ & 0,21 & $\begin{array}{ll}0, L \\
0,02\end{array}$ & 0,03 & 0,21 & 0,41 & $\frac{0,18}{0,18}$ & $\begin{array}{c}0,20 \\
0,20\end{array}$ & 0,23 & 0,19 & 0,12 \\
\hline \multirow{3}{*}{8} & mean & 74,58 & 81,42 & 72,02 & 0,29 & 0,14 & $\begin{array}{l}0,32 \\
\end{array}$ & 85,65 & 123,73 & 132,55 & 33,59 & 6,69 & 132,49 & 95,55 & 65,04 & 105,01 & 159,50 \\
\hline & SD & 20,19 & 21,13 & 19,41 & 0,10 & 0,06 & 0,08 & 22,84 & 2,44 & 2,59 & 8,96 & 2,62 & 26,22 & 18,14 & 17,63 & 18,46 & 22,48 \\
\hline & CV & 0,27 & 0,26 & 0,27 & 0,34 & 0,41 & 0,26 & 0,27 & 0,02 & 0,02 & 0,27 & 0,39 & 0,20 & 0,19 & 0,27 & 0,18 & 0,14 \\
\hline
\end{tabular}

IRITIE Vol. 5, No. 2, 2017 ISSN 1314-8788 (print), ISSN 1314-8796 (online), doi: 10.15547/artte.2017.02.003 




\begin{tabular}{|c|c|c|c|c|c|c|c|c|c|c|c|c|c|c|c|c|c|}
\hline \multirow{3}{*}{9} & mean & 82,36 & 85,07 & 71,89 & 0,21 & 0,16 & 0,34 & 90,70 & 124,89 & 135,33 & 35,57 & 8,16 & 114,78 & 83,16 & 63,77 & 110,20 & 156,33 \\
\hline & SD & 12,38 & 11,76 & 11,20 & 0,05 & 0,05 & 0,05 & 12,73 & 1,48 & 2,73 & 4,99 & 2,54 & 13,83 & 11,96 & 8,46 & 15,25 & 12,11 \\
\hline & CV & 0,15 & 0,14 & 0,16 & 0,26 & 0,33 & 0,14 & 0,14 & 0,01 & 0,02 & 0,14 & 0,31 & 0,12 & 0,14 & 0,13 & 0,14 & 0,08 \\
\hline \multirow{3}{*}{10} & mean & 81,67 & 83,71 & 70,25 & 0,20 & 0,18 & 0,33 & 89,03 & 125,17 & 135,63 & 34,92 & 8,39 & 112,10 & 83,89 & 66,59 & 113,12 & 157,70 \\
\hline & SD & 20,49 & 20,76 & 20,04 & 0,06 & 0,08 & 0,08 & 22,49 & 1,87 & 3,08 & 8,82 & 2,98 & 18,13 & 17,50 & 16,96 & 22,42 & 21,33 \\
\hline & $\mathrm{CV}$ & 0,25 & 0,25 & 0,29 & 0,31 & $\begin{array}{l}, 44 \\
0,44\end{array}$ & 0,24 & 0,25 & 0,01 & 0,02 & 0,25 & 0,36 & 0,16 & 0,21 & 0,25 & 0,20 & 0,14 \\
\hline \multirow{3}{*}{11} & mean & 88,04 & 90,45 & 75,39 & 0,21 & 0,18 & 0,36 & 96,14 & 124,87 & 136,41 & 37,70 & 9,36 & 114,02 & 80,24 & 61,91 & 111,80 & 150,33 \\
\hline & SD & 20,72 & 21,01 & 20,63 & 0,07 & 0,09 & 0,08 & 22,42 & 2,07 & 4,19 & 8,79 & 3,84 & 17,29 & 16,92 & 16,03 & 23,79 & 22,06 \\
\hline & CV & 0,24 & 0,23 & 0,27 & 0,32 & 0,51 & 0,23 & 0,23 & 0,02 & 0,03 & 0,23 & 0,41 & 0,15 & 0,21 & 0,26 & 0,21 & 0,15 \\
\hline \multirow{3}{*}{12} & mean & 91,67 & 95,46 & 79,59 & 0,22 & 0,18 & 0,38 & 101,08 & 124,17 & 136,61 & 39,64 & 9,75 & 115,78 & 79,74 & 57,91 & 110,57 & 144,87 \\
\hline & SD & 20,39 & 21,19 & 20,54 & 0,06 & 0,08 & 0,08 & 22,43 & 2,06 & 3,26 & 8,80 & 2,91 & 16,12 & 16,54 & 16,14 & 21,68 & 22,29 \\
\hline & $\mathrm{CV}$ & 0,22 & 0,22 & 0,26 & 0,30 & 0,43 & 0,22 & 0,22 & 0,02 & 0,02 & 0,22 & 0,30 & 0,14 & 0,21 & 0,28 & 0,20 & 0,15 \\
\hline
\end{tabular}

Table 4. Distances between object areas with cheese and mold

\begin{tabular}{|c|c|c|c|c|c|c|c|c|c|c|c|c|c|c|c|c|c|}
\hline & & \multicolumn{3}{|c|}{ RGB } & \multicolumn{3}{|c|}{ HSV } & \multicolumn{3}{|c|}{ Lab } & \multicolumn{3}{|c|}{$\overline{\mathrm{LCH}}$} & \multicolumn{4}{|c|}{ CMYK } \\
\hline day & & $\mathbf{R}$ & $\mathbf{G}$ & B & $\mathbf{H}$ & $\mathrm{S}$ & V & $\mathbf{L}$ & $\mathbf{a}$ & b & $\mathbf{L}$ & C & $\mathbf{H}$ & C & M & $\mathbf{Y}$ & $K$ \\
\hline \multirow{6}{*}{3} & Mah & 1,8 & 1,8 & 1,8 & 1,7 & 1,8 & 1,8 & 1,8 & 1,7 & 1,8 & 1,8 & 1,8 & 1,7 & 1,8 & 1,8 & 1,8 & 1,8 \\
\hline & $\mathrm{Eu}$ & 25,9 & 23,4 & 21,8 & 0,2 & 0,1 & 0,1 & 24,4 & 1,9 & 4,1 & 9,6 & 4,1 & 52,9 & \begin{tabular}{|l|}
16,6 \\
\end{tabular} & 11,4 & 24,6 & 25,2 \\
\hline & $\mathrm{Cb}$ & 30,4 & 27,7 & 26,3 & 0,2 & 0,1 & 0,1 & 28,7 & 2,3 & 4,9 & 11,3 & 4,8 & 55,7 & 19,5 & 13,5 & 29,1 & 29,6 \\
\hline & Mink & 25,9 & 23,4 & 21,8 & 0,2 & 0,1 & 0,1 & 24,4 & 1,9 & 4,1 & 9,6 & 4,1 & 52,9 & \begin{tabular}{|c|}
16,6 \\
\end{tabular} & 11,4 & 24,6 & 24,6 \\
\hline & Cheb & 24,9 & 22,3 & 20,5 & 0,2 & 0,1 & 0,1 & 23,4 & 1,7 & 3,9 & 9,2 & 3,9 & 52,6 & 15,9 & 10,9 & 23,5 & 24,1 \\
\hline & FDR & 0,6 & 1,7 & 11,9 & 0,5 & 43,8 & 0,6 & 1,6 & 0,3 & 39,9 & 1,6 & 39,4 & 0,2 & 0,7 & 5,4 & 29,7 & 0,7 \\
\hline \multirow{6}{*}{4} & Mah & 1,8 & 1,8 & 1,8 & 1,8 & 1,7 & 1,8 & 1,8 & 1,7 & 1,8 & 1,8 & 1,8 & 1,8 & 1,8 & 1,7 & 1,7 & 1,8 \\
\hline & $\mathrm{Eu}$ & 33,6 & 31,0 & 29,2 & 0,0 & 0,1 & 0,1 & 32,5 & 2,3 & 5,4 & 12,7 & 5,3 & 8,3 & 23,3 & 17,6 & 32,2 & 32,5 \\
\hline & $\mathrm{Cb}$ & 40,5 & 37,4 & 35,5 & 0,0 & 0,1 & 0,2 & 38,8 & 2,8 & 6,5 & 15,2 & 6,4 & 10,1 & 26,6 & 20,8 & 37,9 & 39,0 \\
\hline & Mink & 33,6 & 31,0 & 29,2 & 0,0 & 0,1 & 0,1 & 32,5 & 2,3 & 5,4 & 12,7 & 5,3 & 8,3 & 23,3 & 17,6 & 32,2 & 32,2 \\
\hline & Cheb & 31,7 & 29,3 & 27,5 & 0,0 & 0,1 & 0,1 & 30,8 & 2,1 & 5,1 & 12,1 & 5,0 & 7,7 & 22,7 & 16,8 & 30,7 & 30,8 \\
\hline & FDR & 4,4 & 3,1 & 3,0 & 5,0 & 0,0 & 4,5 & 3,2 & 7,0 & 0,3 & 3,2 & 0,2 & 6,0 & 4,0 & 0,6 & 1,5 & 3,4 \\
\hline \multirow{6}{*}{5} & Mah & 1,8 & 1,8 & 1,8 & 1,8 & 1,7 & 1,8 & 1,8 & 1,8 & 1,7 & 1,8 & 1,7 & 1,7 & 1,8 & 1,8 & 1,8 & 1,8 \\
\hline & $\mathrm{Eu}$ & 33,8 & 34,4 & 36,8 & 0,0 & 0,2 & 0,1 & 35,5 & 3,1 & 10,0 & 13,9 & 9,7 & 15,0 & 22,1 & 24,3 & 49,7 & 35,9 \\
\hline & $\mathrm{Cb}$ & 40,0 & 40,9 & 44,0 & 0,1 & 0,2 & 0,2 & 41,8 & 3,8 & 11,6 & 16,4 & 11,3 & 17,2 & 25,0 & 28,6 & 57,8 & 42,1 \\
\hline & Mink & 33,8 & 34,4 & 36,8 & 0,0 & 0,2 & 0,1 & 35,5 & 3,1 & 10,0 & 13,9 & 9,7 & 15,0 & 22,1 & 24,3 & 49,7 & 49,7 \\
\hline & Cheb & 32,2 & 32,7 & 34,9 & 0,0 & 0,2 & 0,1 & 33,9 & 2,9 & 9,6 & 13,3 & 9,4 & 14,5 & 21,5 & 23,2 & 47,8 & 34,3 \\
\hline & FDR & 3,9 & 2,4 & 1,5 & 1,4 & 0,0 & 3,5 & 2,5 & 2,4 & 0,3 & 2,5 & 0,2 & 1,4 & 4,2 & 0,5 & 0,4 & 2,6 \\
\hline \multirow{6}{*}{6} & Mah & 1,8 & 1,8 & 1,7 & 1,8 & 1,6 & 1,8 & 1,7 & 1,8 & 1,7 & 1,7 & 1,7 & 1,8 & 1,8 & \begin{tabular}{l|}
1,7 \\
\end{tabular} & 1,7 & 1,8 \\
\hline & $\mathrm{Eu}$ & 20,5 & 21,4 & 26,0 & 0,1 & 0,1 & 0,1 & 22,1 & 3,1 & 8,0 & 8,7 & 7,0 & 33,2 & 16,4 & 18,0 & 40,0 & 22,5 \\
\hline & $\mathrm{Cb}$ & 25,0 & 26,2 & 32,1 & 0,1 & 0,2 & 0,1 & 26,7 & 3,8 & 9,8 & 10,5 & 8,6 & 37,5 & 18,7 & 22,1 & 48,6 & 27,3 \\
\hline & Mink & 20,5 & 21,4 & 26,0 & 0,1 & 0,1 & 0,1 & 22,1 & 3,1 & 8,0 & 8,7 & 7,0 & 33,2 & 16,4 & 18,0 & 40,0 & 40,0 \\
\hline & Cheb & 19,0 & 20,0 & 24,1 & 0,1 & 0,1 & 0,1 & 20,7 & 2,8 & 7,4 & 8,1 & 6,5 & 32,4 & 15,9 & 16,8 & 37,4 & 21,0 \\
\hline & FDR & 21,4 & 7,7 & 2,7 & 4,0 & 0,7 & 16,4 & 9,3 & 16,6 & 2,3 & 9,3 & 2,4 & 5,4 & 17,9 & 0,0 & 0,0 & 12,0 \\
\hline & Mah & 1,8 & 1,8 & 1,7 & 1,8 & 1,6 & 1,8 & 1,8 & 1,8 & 1,7 & 1,8 & 1,7 & 1,8 & 1,8 & 1,7 & 1,7 & 1,8 \\
\hline & $\mathrm{Eu}$ & 24,5 & 23,2 & 23,5 & 0,1 & 0,1 & 0,1 & 24,2 & 3,3 & 6,0 & 9,5 & 5,3 & 30,1 & 21,8 & 16,9 & 28,5 & 23,7 \\
\hline 7 & $\mathrm{Cb}$ & 29,9 & 28,5 & 29,5 & 0,1 & 0,1 & 0,1 & 29,5 & 4,0 & 7,5 & 11,6 & 6,6 & 33,6 & 24,6 & 20,9 & 36,1 & 28,9 \\
\hline 7 & Mink & 24,5 & 23,2 & 23,5 & 0,1 & 0,1 & 0,1 & 24,2 & 3,3 & 6,0 & 9,5 & 5,3 & 30,1 & 21,8 & 16,9 & 28,5 & 28,5 \\
\hline & Cheb & 22,9 & 21,5 & 21,4 & 0,1 & 0,1 & 0,1 & 22,6 & 3,0 & 5,4 & 8,9 & 4,8 & 29,5 & 21,3 & 15,5 & 25,8 & 22,0 \\
\hline & FDR & 22,4 & 9,8 & 4,1 & 5,8 & 3,8 & 20,5 & 11,4 & 21,3 & 10,5 & 11,4 & 10,7 & 8,0 & 14,9 & 0,1 & 0,0 & 15,7 \\
\hline & Mah & 1,8 & 1,8 & 1,8 & 1,7 & 1,7 & 1,8 & 1,8 & 1,8 & 1,7 & 1,8 & 1,8 & 1,7 & 1,8 & 1,7 & 1,8 & 1,8 \\
\hline & $\mathrm{Eu}$ & 25,7 & 26,7 & 26,1 & 0,1 & 0,1 & 0,1 & 28,1 & 3,4 & 4,2 & 11,0 & 4,2 & 28,1 & 21,1 & 21,6 & 26,2 & 27,9 \\
\hline & $\mathrm{Cb}$ & 31,2 & 32,3 & 32,3 & 0,1 & 0,1 & 0,1 & 33,6 & 4,2 & 5,2 & 13,2 & 5,3 & 31,3 & 23,7 & 26,0 & 32,9 & 33,4 \\
\hline 8 & Mink & 25,7 & $\begin{array}{l}26,7 \\
\end{array}$ & 26,1 & 0,1 & 0,1 & 0,1 & 28,1 & 3,4 & 4,2 & 11,0 & 4,2 & 28,1 & 21,1 & 21,6 & 26,2 & 26,2 \\
\hline & Cheb & 24,1 & 25,1 & 24,1 & 0,1 & 0,1 & 0,1 & 26,6 & 3,1 & 3,7 & 10,4 & 3,8 & 27,5 & 20,6 & 20,3 & 23,9 & 26,4 \\
\hline & FDR & 22,0 & 9,3 & 4,4 & 4,0 & 8,8 & 17,8 & 10,3 & 14,7 & 23,6 & 10,3 & 20,5 & 5,1 & 15,8 & 0,1 & 0,0 & 13,6 \\
\hline & Mah & 1,8 & 1,8 & 1,8 & 1,7 & 1,7 & 1,8 & 1,8 & 1,7 & 1,8 & 1,8 & 1,8 & 1,7 & 1,8 & 1,7 & 1,8 & 1,8 \\
\hline & $\mathrm{Eu}$ & 17,6 & 17,4 & 18,6 & 0,1 & 0,1 & 0,1 & 18,0 & 2,5 & 4,5 & 7,1 & 4,4 & 15,3 & 14,2 & 13,5 & 24,6 & 17,5 \\
\hline & $\mathrm{Cb}$ & 21,9 & 21,8 & 23,7 & 0,1 & 0,1 & 0,1 & 22,5 & 3,1 & 5,7 & 8,8 & 5,6 & 17,5 & 16,6 & 17,1 & 31,2 & 21,9 \\
\hline 9 & Mink & 17,6 & 17,4 & 18,6 & 0,1 & 0,1 & 0,1 & 18,0 & 2,5 & 4,5 & 7,1 & 4,4 & 15,3 & 14,2 & 13,5 & 24,6 & 24,6 \\
\hline & Cheb & 16,1 & 15,8 & 16,8 & 0,1 & 0,1 & 0,1 & 16,5 & 2,3 & 4,1 & 6,5 & 4,0 & 14,8 & 13,7 & 12,2 & 22,2 & 16,0 \\
\hline & FDR & 26,7 & 12,5 & 1,0 & 3,0 & 16,3 & 26,1 & 14,4 & 10,6 & 34,1 & 14,4 & 34,6 & 5,1 & 17,6 & 0,1 & 3,4 & 20,9 \\
\hline & Mah & 1,8 & 1,8 & 1,8 & 1,7 & 1,7 & 1,8 & 1,8 & 1,7 & 1,8 & 1,8 & 1,8 & 1,6 & 1,8 & 1,7 & 1,8 & 1,8 \\
\hline & Eu & 27,9 & 29,2 & 31,5 & 0,1 & 0,1 & 0,1 & 30,2 & 3,0 & 5,6 & 11,9 & 5,6 & 18,0 & 20,6 & 22,8 & 36,6 & 28,5 \\
\hline 10 & $\mathrm{Cb}$ & 34,5 & 36,2 & 39,8 & 0,1 & 0,2 & 0,1 & 37,2 & 3,8 & 7,1 & 14,6 & 7,1 & 20,3 & 23,9 & 28,2 & 46,5 & 35,0 \\
\hline 10 & Mink & 27,9 & 29,2 & 31,5 & 0,1 & 0,1 & 0,1 & 30,2 & 3,0 & 5,6 & 11,9 & 5,6 & 18,0 & 20,6 & 22,8 & 36,6 & 36,6 \\
\hline & Cheb & 25,8 & 26,8 & 28,6 & 0,1 & 0,1 & 0,1 & 28,0 & 2,7 & 5,1 & 11,0 & 5,0 & 17,5 & 19,8 & 21,0 & 33,0 & 26,5 \\
\hline & FDR & 12,7 & 6,1 & 0,6 & 1,7 & 7,5 & 11,8 & 6,6 & 5,2 & 27,5 & 6,6 & 26,6 & 2,2 & 9,3 & 0,3 & 1,5 & 9,7 \\
\hline & Mah & 1,8 & 1,8 & 1,8 & 1,8 & 1,7 & 1,8 & 1,8 & 1,7 & 1,8 & 1,8 & 1,7 & 1,8 & 1,7 & 1,7 & 1,8 & 1,8 \\
\hline & $\mathrm{Eu}$ & 27,7 & 29,2 & 33,0 & 0,1 & 0,1 & 0,1 & 29,7 & 2,9 & 7,0 & 11,6 & 6,7 & 20,1 & 19,2 & 20,9 & 37,3 & 29,0 \\
\hline 11 & $\mathrm{Cb}$ & 34,4 & 36,7 & 42,1 & 0,1 & 0,2 & 0,1 & 36,9 & 3,6 & 8,9 & 14,5 & 8,5 & 22,7 & 21,8 & 25,9 & 47,2 & 35,8 \\
\hline 11 & Mink & 27,7 & 29,2 & 33,0 & 0,1 & 0,1 & 0,1 & 29,7 & 2,9 & 7,0 & 11,6 & 6,7 & 20,1 & 19,2 & 20,9 & 37,3 & 37,3 \\
\hline & Cheb & 25,5 & 26,6 & 29,6 & 0,1 & 0,1 & 0,1 & 27,4 & 2,7 & 6,4 & 10,7 & 6,1 & 19,6 & 18,7 & 19,2 & 33,7 & 26,8 \\
\hline & FDR & 23,3 & 11,0 & 4,3 & 2,9 & 2,4 & 21,1 & 12,1 & 17,2 & 8,4 & 12,1 & 9,3 & 5,1 & 14,3 & 0,2 & 0,0 & 17,5 \\
\hline & Mah & 1,8 & 1,8 & 1,8 & 1,7 & 1,7 & 1,8 & 1,8 & 1,8 & 1,8 & 1,8 & 1,8 & 1,8 & \begin{tabular}{ll|}
1,7 \\
\end{tabular} & $\begin{array}{l}1,7 \\
\end{array}$ & 1,8 & 1,8 \\
\hline & $\mathrm{Eu}$ & 28,9 & 31,7 & 34,9 & 0,1 & 0,1 & 0,1 & 31,7 & 3,3 & 6,3 & 12,5 & 6,1 & 18,7 & 19,3 & 24,5 & 39,0 & 31,0 \\
\hline & $\mathrm{Cb}$ & 36,3 & 40,2 & 44,5 & 0,1 & 0,2 & 0,1 & 40,0 & 4,1 & 8,0 & 15,7 & 7,6 & 21,2 & 22,7 & 31,0 & 49,6 & 38,9 \\
\hline 12 & Mink & 28,9 & 31,7 & 34,9 & 0,1 & 0,1 & 0,1 & 31,7 & 3,3 & 6,3 & 12,5 & 6,1 & 18,7 & 19,3 & 24,5 & 39,0 & 39,0 \\
\hline & Cheb & 26,4 & 28,6 & 31,3 & 0,1 & 0,1 & 0,1 & 28,9 & 2,9 & 5,7 & 11,3 & 5,5 & 18,2 & 18,4 & 22,1 & 35,2 & 28,3 \\
\hline & FDR & 12,1 & 3,3 & 0,6 & 3,5 & 5,2 & 10,3 & 4,5 & 21,2 & 13,4 & 4,5 & 14,6 & 6,8 & 10,9 & 0,4 & 0,5 & 7,7 \\
\hline
\end{tabular}

The results from distance functions calculated are presented in Table 4. These distances are between the values of color components of object areas with cheese and mold and their change in storage period. Changes of the distances are shown in days from 3 to 12 for every color component from 5 color models.

IRTTIE Vol. 5, No. 2, 2017 ISSN 1314-8788 (print), ISSN 1314-8796 (online), doi: 10.15547/artte.2017.02.003 


\section{IRTITE}

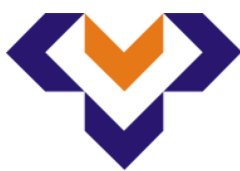

Ipplied Reseirlches in Technics, Technologies and Bductition

Journal of the Faculty of Technics and Technologies, Trakia University https:///sites.google.com/a/trakia-uni.bg/artte/

The resulting distances are processed with method "Correspondence analysis. Figure 3 presents the results from correspondence analysis of the data. The influence of three parameters are present. On $X$ axis are present distances between color components, $Y$ axis is for color components and $Z$ axis for days of storage. The color components have higher influence $95 \%$ on the change of data, distance functions have $5 \%$ and days of storage $0,3 \%$.
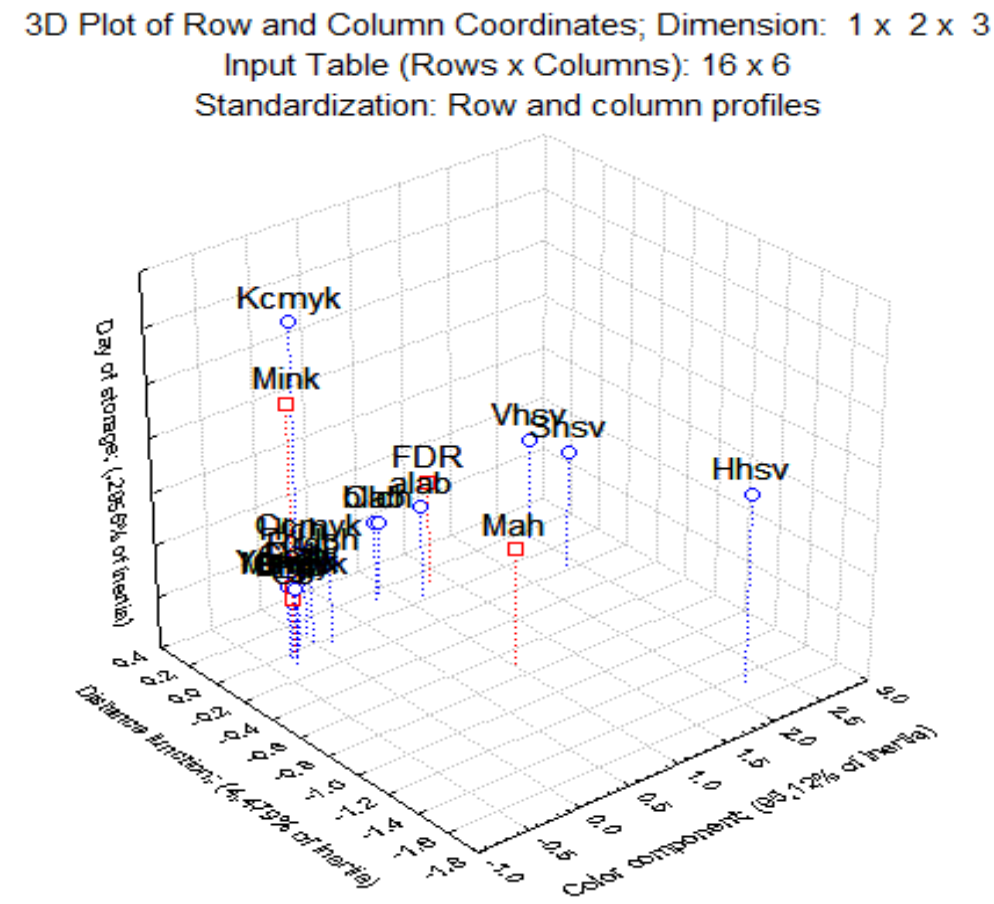

Figure 3. Results from correspondence analysis

Table 5. Results from correspondence analysis

\begin{tabular}{|c|c|c|c|c|c|c|}
\hline $\begin{array}{l}\text { Distance } \\
\text { function }\end{array}$ & Mah & Eu & $\mathrm{Cb}$ & Mink & Cheb & FDR \\
\hline $\begin{array}{c}\text { Color } \\
\text { component }\end{array}$ & $\mathrm{H}(\mathrm{HSV})$ & $\begin{array}{c}\text { R (RGB) } \\
\text { G (RGB) } \\
\text { B (RGB) } \\
\text { L (Lab) } \\
\text { L (LCH) } \\
\text { H (LCH) } \\
\text { C (CMYK) } \\
\text { M (CMYK) } \\
\text { Y (CMYK) }\end{array}$ & $\begin{array}{c}\mathrm{R}(\mathrm{RGB}) \\
\mathrm{G}(\mathrm{RGB}) \\
\mathrm{B}(\mathrm{RGB}) \\
\mathrm{L}(\mathrm{Lab}) \\
\mathrm{L}(\mathrm{LCH}) \\
\mathrm{H}(\mathrm{LCH}) \\
\mathrm{C}(\mathrm{CMYK}) \\
\mathrm{M}(\mathrm{CMYK}) \\
\mathrm{Y}(\mathrm{CMYK}) \\
\end{array}$ & $\mathrm{K}(\mathrm{CMYK})$ & $\begin{array}{c}\mathrm{R}(\mathrm{RGB}) \\
\mathrm{G}(\mathrm{RGB}) \\
\mathrm{B}(\mathrm{RGB}) \\
\mathrm{L}(\mathrm{Lab}) \\
\mathrm{L}(\mathrm{LCH}) \\
\mathrm{H}(\mathrm{LCH}) \\
\mathrm{C}(\mathrm{CMYK}) \\
\mathrm{M}(\mathrm{CMYK}) \\
\mathrm{Y}(\mathrm{CMYK})\end{array}$ & $\begin{array}{l}\text { S (HSV) } \\
\text { V (HSV) } \\
\text { a (Lab) } \\
\text { b (Lab) } \\
\text { C (LCH) }\end{array}$ \\
\hline
\end{tabular}

Mah - Mahalanobis distance; Eu - Euclidean distance; $\mathrm{Cb}$ - distance of Manhattan; Mink - Mincowski distance; Cheb - Chebyshev distance; FDR - Fisher discriminant ratio

The results obtained from correspondence analysis are systematized in Table 5. By Mahalanobis and Minkowski distances the object areas with cheese and mold can be separated only with one color component H (HSV) and K (CMYK). Results for measured distances between color components by Fisher discriminant ratio show that 5 color components can be used for separation of object areas on the surface of cheese in storage period. Most of the color components -9 can be used to separate object areas by Euclidean, Chebyshev and Manhattan distances.

IRTIIE Vol. 5, No. 2, 2017 ISSN 1314-8788 (print), ISSN 1314-8796 (online), doi: 10.15547/artte.2017.02.003 


\section{IRTITE}

The results from analysis of distances between color components are used for classification. Four of the color components with higher distances are used - S (HSV), V (HSV), L (LCH) and $\mathrm{Y}(\mathrm{CMYK})$.

Figure 4 represents examples of classification of object area with cheese and mold with cluster classifier. Figure 5 represents examples of classification of object area with cheese and mold by non-linear discriminant analysis. On figures are shown used color components, day of storage and classification error.

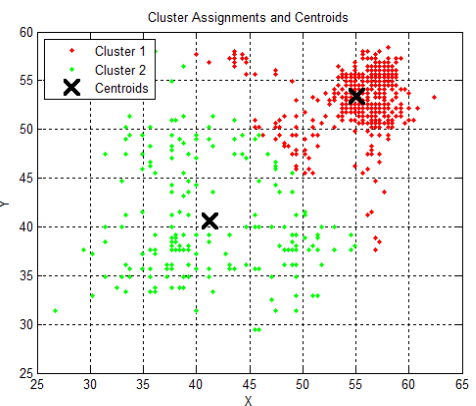

a) Day 3, L ( $\mathrm{LCH}), e=40 \%$

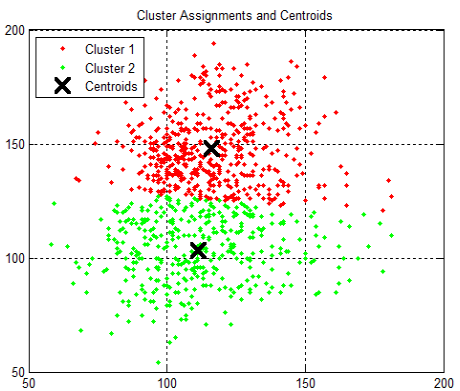

b) Day 12, Y (CMYK), $\mathrm{e}=12 \%$

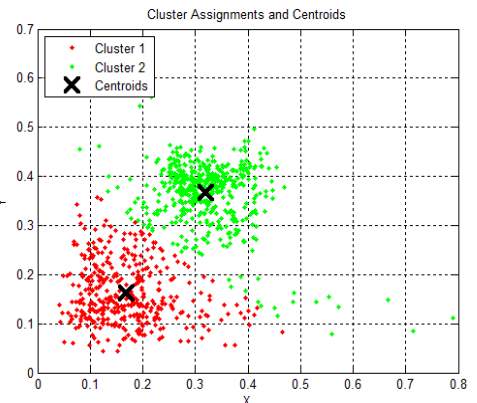

c) Day $11, \mathrm{~S}(\mathrm{HSV})$, $\mathrm{e}=14 \%$

Figure 4.

Examples of classification with Cluster analysis by color components of object areas

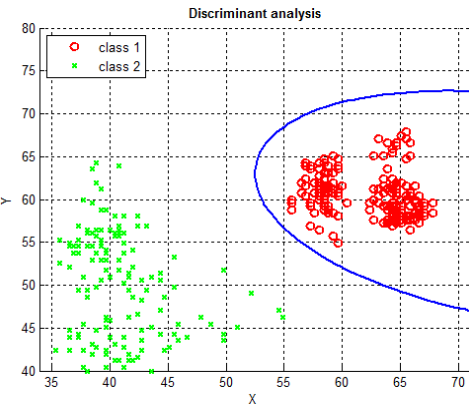

a) Day $5, \mathrm{~L}(\mathrm{LCH}), \mathrm{e}=1 \%$

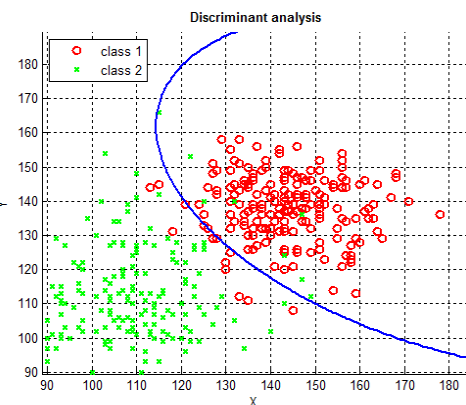

b) Day 9, Y (CMYK), $\mathrm{e}=6 \%$



c) Day $5, \mathrm{~S}(\mathrm{HSV}), \mathrm{e}=1 \%$

Figure 5. Examples of classification with discriminant analysis by color components of object areas

Table 6. Results from classification

\begin{tabular}{|r|r|r|r|r|r|r|r|r|}
\hline & \multicolumn{4}{|c|}{ Cluster analysis } & \multicolumn{5}{|c|}{ Discriminant analysis } \\
\hline Day & S (HSV) & V (HSV) & L (LCH) & Y (CMYK) & S (HSV) & V (HSV) & L (LCH) & Y (CMYK) \\
\hline 3 & $1 \%$ & $44 \%$ & $39 \%$ & $1 \%$ & $1 \%$ & $13 \%$ & $6 \%$ & $1 \%$ \\
\hline 4 & $80 \%$ & $5 \%$ & $20 \%$ & $60 \%$ & $17 \%$ & $1 \%$ & $1 \%$ & $2 \%$ \\
\hline 5 & $79 \%$ & $12 \%$ & $21 \%$ & $78 \%$ & $1 \%$ & $1 \%$ & $1 \%$ & $21 \%$ \\
\hline 6 & $20 \%$ & $1 \%$ & $1 \%$ & $88 \%$ & $1 \%$ & $1 \%$ & $1 \%$ & $29 \%$ \\
\hline 7 & $5 \%$ & $1 \%$ & $1 \%$ & $48 \%$ & $1 \%$ & $1 \%$ & $1 \%$ & $28 \%$ \\
\hline 8 & $2 \%$ & $1 \%$ & $1 \%$ & $42 \%$ & $1 \%$ & $1 \%$ & $1 \%$ & $32 \%$ \\
\hline 9 & $1 \%$ & $1 \%$ & $1 \%$ & $3 \%$ & $1 \%$ & $1 \%$ & $1 \%$ & $6 \%$ \\
\hline 10 & $2 \%$ & $1 \%$ & $1 \%$ & $17 \%$ & $1 \%$ & $1 \%$ & $1 \%$ & $19 \%$ \\
\hline 11 & $14 \%$ & $1 \%$ & $1 \%$ & $31 \%$ & $6 \%$ & $1 \%$ & $1 \%$ & $21 \%$ \\
\hline 12 & $3 \%$ & $1 \%$ & $1 \%$ & $12 \%$ & $2 \%$ & $1 \%$ & $1 \%$ & $29 \%$ \\
\hline min & $1 \%$ & $1 \%$ & $1 \%$ & $1 \%$ & $1 \%$ & $1 \%$ & $1 \%$ & $1 \%$ \\
\hline max & $80 \%$ & $44 \%$ & $39 \%$ & $88 \%$ & $17 \%$ & $13 \%$ & $6 \%$ & $32 \%$ \\
\hline
\end{tabular}

IRTIIE Vol. 5, No. 2, 2017 ISSN 1314-8788 (print), ISSN 1314-8796 (online), doi: 10.15547/artte.2017.02.003 


\section{ARTTIE Y}

Ipplied Reseitrones in Technics, Technologies and Bductition

Journal of the Faculty of Technics and Technologies, Trakia University https://sites.google.com/a/trakia-uni.bg/artte/

The results of analysis of classification errors for the two compared classifiers are presented on Table 6 . Cluster analysis show higher classification errors reaching $80 \%$. Only by V (HSV) color component the error is under $5 \%$ for values of this color component in 6 to 11 day of storage. Lower classification error is observed for non-linear discriminant classifier. The maximum error is $32 \%$ for $\mathrm{Y}$ (CMYK) color component. For other color components the classification error is $6-13 \%$ using S (HSV), V (HSV) and L (LCH). These results show that non-linear discriminant analysis is suitable for separation of object areas with cheese and mold by color features in storage period.

\section{CONCLUSION}

The article offers a comparative analysis of criteria for determining the informability of color features for separating object areas on the surface of white brined cheese during the storage period. The color characteristics of the object areas depend on a great many factors. Even within the field of a certain type, for example an area of mold, even within a sample, the color characteristics noticeably vary in non-small range. In addition, they vary depending on the duration of sample storage, storage conditions, and other factors such as ambient temperature and humidity. Compared to the refrigeration conditions prescribed by the manufacturer where these changes occur after the expiry date of the product at room temperature, these changes occur on the third and fourth day of storage of the product.

Established is the possibility of reducing the number of features through distance functions. In practice, it can be reduced, depending on the function being applied.

Four color components S (HSV), V (HSV), L (LCH) and Y (CMYK) are defined as informative by Euclidean, Chebyshev and Manhattan distances. These color components are used for assessment of the classification error of two classifiers - cluster analysis and discriminant function analysis. The results from analysis show that color components S (HSV), V (HSV) and $\mathrm{L}(\mathrm{LCH})$ are suitable for separation of object areas with white brined cheese and mold in storage period.

\section{ACKNOWLEDGMENTS}

The work on this report is related to the research project 3.FTT/ 30.05.2016 "Contactless methods for evaluation of main quality characteristics of dairy products".

\section{REFERENCES}

[1] Baycheva S., Application of devices of measurement of color in analysis of food products, Innovation and entrepreneurship - Applied scientific journal, Vol.4, No.4, 2016, ISSN 1314-9253, pp. 43-59.

[2] BNS 15:2010, Bulgarian white cheese in brine (in Bulgarian).

[3] BNS 15:2010/Amendment 1:2014, Bulgarian white cheese in brine - Amendment 1:2014 (in Bulgarian).

[4] Discriminant function analysis, https://www.mathworks.com/help/stats/discriminantanalysis.html (available on 03.05.2017).

[5] Georgieva K., E. Kirilova, Ts. Georgieva, P. Daskalov, Selection of informative color features complexes from digital images of healthy and diseased vine leaves, ARTTE Vol. 3, No. 4, 2015, ISSN 1314-8796, pp. 289-295.

[6] Kazlacheva Z., J. Ilieva, M. Zhekova, P. Dineva, Fashion design on the base of connection between colors and lines, ARTTE Vol. 2, No. 1, 2014, ISSN 1314-8796, pp.54-64. 


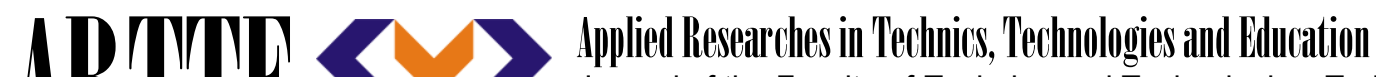 Journal of the Faculty of Technics and Technologies, Trakia University https://sites.google.com/a/trakia-uni.bg/artte/}

[7] k-means clustering, https://www.mathworks.com/help/stats/kmeans.html (available on 11.06.2017).

[8] Mladenov M., St. Penchev, M. Dejanov, Complex assessment of food products quality using analysis of visual images, spectrophotometric and hyperspectral characteristics, International Journal of Engineering and Innovative Technology (IJEIT), Vol. 4, 2015, ISSN 2277-3754, pp. 23-32.

[9] Statsoft Statistica, http://www.statsoft.com (available on 17.06.2017)

[10] Taneva I., N. Petkova, I. Dimov, I. Ivanov, P. Denev, Characterization of Rose Hip (Rosa canina L.) Fruits Extracts and Evaluation of Their in vitro Antioxidant Activity, Journal of Pharmacognosy and Phytochemistry, vol. 5, No. 2, 2016, pp. 35-38. 